\title{
Emerging Technologies for Heart Diseases
}

\author{
Makoto Ohta ${ }^{\mathrm{a}, \mathrm{b}}$ \\ ${ }^{a}$ Institute of Fluid Science, Tohoku University, Sendai, 980-8577, Japan \\ ${ }^{\mathrm{b}}$ ELyTMax UMI 3757, CNRS, Université de Lyon-Tohoku University, International Joint Unit, Tohoku \\ University, Sendai, 980-8577, Japan \\ E-mail: makoto.ohta@tohoku.ac.jp
}

Books editor: Udi Nussinovitch MD PhD, Publisher. Elsevier, Academic Press, ISBN: 978-0-12-813706-2 and 978-0-12-813704-8, Hardback: 1064 pages

The two volumes of "Emerging Technologies for Heart Diseases" edited by Udi Nussinovitch, MD, $\mathrm{PhD}$ from the Department of Cardiology and Applicative Cardiovascular Research Center (ACRC) at Meir Medical Center, Kfar Saba, Israel, are released. Volume 1 focuses on heart failure and valvular disorders whereas the second volume covers treatments for myocardial ischemia and arrhythmias.

Among other topics, the books elaborate on emerging therapies in coronary balloon angioplasty, stenting and bioabsorbable scaffolds. Treatments for and the prevention of tachyarrhythmias based on the cardiac ablation therapy, gene and cell therapy including optogenetics, or molecular therapies are also extensively discussed. The ninth section introduces cardiac monitoring using flexible electronics, whereas the tenth section focuses on the prevention of cerebral embolization. Also, several chapters are devoted to minimally-invasive methods for treatment of structural heart abnormalities, and novel treatments for hypertension. The books cover all medical devices related to cardiovascular therapeutics, and list so many cardiovascular technologies, enough to be called "an encyclopedia". Therefore, this book is useful for clinicians of the cardiovascular field and for endovascular surgeons, radiologists or radiographers, and also for cardiovascular researchers.

The perspective of technological advancements and future outlook, as well a clear overview on current scientific data, may enable clinicians to use the medical devices more effectively. Based on the information provided in the books, clinicians can better collaborate with biomedical engineers to create a new generation of these medical devices.

The language is scientific and medical, but not overly technical, and so anyone can read this book. Potentially, clinicians can also encourage their patients to read it from the viewpoint of informed consent.

In my opinion, this book is an appropriate read for all researchers and students, especially for those who focus on biomedical engineering. The advantage of this book is also evident by its ability to explain medical technology to non-clinicians. This will enhance the conversation between individuals involved in the biomedical field. The book will also enhance the understanding of clinicians as to biomedical engineering aspects including physics, chemicals and biologicals.

It is also noticed that the text found in the books is supported by many updated references. These references are also a good starting point for anyone who wishes to dive deeper into the reviewed cardiovascular fields. 
The historical aspects of medical device development, presented in the books, may be useful and encourage researchers to create the next generation of modifications for medical devices. The books contain an interesting overview of how medical devices became smaller, more complex and utilized more combination technologies.

In conclusion, I am sure that this book will be useful for all those who work in the cardiovascular field, both clinicians and non-clinicians. 